\title{
Application of modified complex Tremblay operator
}

\begin{abstract}
In this paper, we introduce a new fractional integral operator defined by modified fractional derivative Tremblay operator of analytic functions and show that the univalence of this integral operator is preserved under certain sufficient conditions in complex domain $\mathbb{C}$.
\end{abstract}

Keyword: Tremblay operator; Fractional integral operator 